\title{
Comparison of prolactin, follicle-stimulating hormone, luteinizing hormone, estradiol, thyroid- stimulating hormone, free thyroxine and body mass index between infertile and fertile Saudi women
}

\begin{abstract}
The objective of this study was to compare the levels of prolactin, FSH, LH, E2, TSH, FT4 and BMI between infertile Saudi women with high prolactin and fertile Saudi women with normal prolactin. The study individuals were divided into two groups; infertile Saudi women with high prolactin (Group 1) and fertile Saudi women with normal prolactin (Group 2). This study used the ARCHITECT i1000SR immunoassay analyzer for the assessment of all serum hormones. The prolactin in Group 1 was higher than in the Group 2. The FSH and LH values were similar in both groups. The TSH in Group 1 was higher than in the Group 2. The FT4 values were similar in both groups. The BMI in both groups was greater than 25 (all patients were in over weight category). In Group 1, 40\% women were over weight and in Group 2, $39 \%$ women were over weight. The rate of failed cycles in group 1 was higher than in Group 2, also cases of PCOS in Group 1 were more than in the Group 2. The rate of positive pregnancy in Group 1 is lower than in the Group 2. The results of this study demonstrated that increased levels of prolactin and TSH are among the causes of infertility in Saudi women.
\end{abstract}

Keywords: prolactin, TSH, infertile, Saudi women
Volume 12 Issue 2 - 202 I

\author{
Alyaa Abdulrahman Asiel Elnour, ${ }^{1,3}$ Murid \\ Javed, ${ }^{2}$ Murwan Khalid Sabah Elkhier,' Ayman \\ Salih Omer Idris,' Wala Mohamed Mahmoud \\ Salih,' Hamad Al Sufyan² \\ Department of Biochemistry and Molecular Biology, Faculty of \\ Science and Technology, AI Neelain University, Sudan \\ ${ }^{2}$ Embryology and Andrology Laboratories, Thuriah Medical \\ Centre, Saudi Arabia \\ ${ }^{3}$ Hormone Analysis Laboratory, Thuriah Medical Centre, Saudi \\ Arabia
}

Correspondence: Murid Javed, Embryology and Andrology Laboratories, Thuriah Medical Centre, Riyadh, Saudi Arabia, Email murid.javed@gmail.com

Received: April 14,202I | Published: April 27, 202 I

\section{Introduction}

Infertility is a global problem affecting people around the world. Its causes and intensity may vary according to the geographical location and socio-economic conditions. According to the statistics, annually 60-80 million couples around the world suffer from infertility. ${ }^{1}$ The term primary infertility is used when a woman has never conceived and the secondary infertility is the inability to conceive after at least one successful conception in the past. ${ }^{2}$ Infertility places a huge psychological burden on infertile couples, especially for women. Greater knowledge of the factors affecting fertility may help to decrease the incidence of infertility by allowing couples to avoid certain risk factors. In Saudi society, infertility is not only a medical but also a social problem as cultural customs may equate infertility with a failure on a personal, interpersonal or social level. ${ }^{3}$

Apart from medical issues, age, smoking, drinking, increased body mass index (BMI), lifestyle and environmental factors are considered major risk factors leading to infertility. ${ }^{4}$ Hormonal disturbances have also been considered of great importance for causes and diagnosis of female infertility. An increase in FSH in women may indicate a reduction in the production of good quality eggs. A woman's chances for pregnancy may be lower than expected for her age. However, it does not mean she has no chance of conceiving. She may have more difficulty conceiving and may require infertility treatment. ${ }^{5}$ The hormonal infertility is a growing problem among women. The hyperprolactinemia is one of the important factors in pathogenesis of anovulation, amenorrhea, galactorrhea and other menstrual disorders. Hypothyroidism may also cause failure to ovulation in women of reproductive age. ${ }^{6}$ Prolactin is synthesized by the lactotrophs in anterior pituitary and is regulated by the release of dopamine from the substantia nigra, ventral tegmental area, and hypothalamus of the brain and acts as prolactin inhibitory factor. ${ }^{7}$ The main function of prolactin is milk synthesis and maintenance of milk secretion during pregnancy and lactation and the development of mammary glands. Serum prolactin levels rise rapidly during pregnancy with increase in the size and number of lactotrophs. During lactation suckling induces rapid secretion of prolactin via a neuroendocrine reflex pathway. In the absence of pregnancy, hyperprolactinemia may present with symptoms of hypogonadotropic hypogonadism, menstrual disturbances and infertility. ${ }^{8}$

Several hormones and neuropeptides have been suggested to promote PRL release including thyrotropin-releasing hormone (TRH), endogenous opioids, oxytocin, serotonin, vasopressin, vasoactive intestinal polypeptide (VIP), neurotensin, galanin, and salsolinol, ${ }^{9,10}$ epithelial growth factor, estrogens, and histidine. ${ }^{11}$ Hypothyroidism stimulates increased secretion of TRH which stimulates thyrotrophs and lactotrophs, causing increase in the levels of both TSH and prolactin. ${ }^{12}$ Polycystic ovary syndrome (PCOS) is the most frequent endocrinopathy, affecting up to $10 \%$ women of reproductive age, It is characterized by ovulatory dysfunction, hyperandrogenism, and metabolic changes. ${ }^{13}$ The objective of this study was to compare the levels of prolactin, TSH, FSH, LH, E, FT4 and BMI among infertile and fertile women in Riyadh, Saudi Arabia.

\section{Materials and methods}

\section{Study design}

A hospital-based case control study was carried-out on fertile and infertile women who were residents of Al Riyadh State, Kingdom of Saudi Arabia from February 2018 to December 2019. The fertile women served as control. 


\section{Study population}

One hundred infertile women with history of primary or secondary female factor infertility served as study group (Group 1). Their ages ranged from 18-40years. One hundred females in their reproductive age, who were not suffering from infertility or any reproductive endocrine disorder, were selected to serve as the control group (Group 2). Their ages ranged from 18 to 40years. The investigations were carried out from February 2018 to December 2019 at Thuriah Medical Centre in Riyadh, Saudi Arabia.

\section{Sampling and data collection}

About $5 \mathrm{ml}$ of venous blood was collected on the second day of the menstrual period from each woman. The blood was centrifuged at $3000 \mathrm{rpm}$ for $15 \mathrm{~min}$ to obtain the serum which was used for measurement of study hormones.

\section{Study parameters}

The study parameters were; serum prolactin (PRL), follicle stimulating hormone (FSH), luteinizing hormone ( $\mathrm{LH})$, Estradiol $\left(\mathrm{E}_{2}\right)$, thyroid-stimulating hormone (TSH), free thyroxine (FT4) and body mass index (MBI). The hormonal assessments were carried out using appropriate enzyme-linked immune sorbent assays by the ARCHITECT i1000SR immunoassay analyzer (Abbott Core Laboratory).

\section{Statistical analysis}

This study analyzed the prevalence of hyperprolactinemia among infertile women, its potential socio-demographic risk factors and its clinical and biochemical parameters. The SPSS version 21 was used for statistical analysis. Categorical data were analyzed by chi square test and $\mathrm{p}$ value $<0.05$ was considered statistically significant. The descriptive statistics was calculated for study variables. The independent student t-test was used to compare the study groups. Correlation was calculated to study the association between different variables.

\section{Results and discussion}

A total of 200 women were included in the study. Out of 200 enrolled participants, 100 (Group 1) had primary infertility. One hundred age matched apparently healthy fertile women were enrolled as control (Group 2). There was no significant difference of body mass index between Group 1 and 2 (Figure 1).

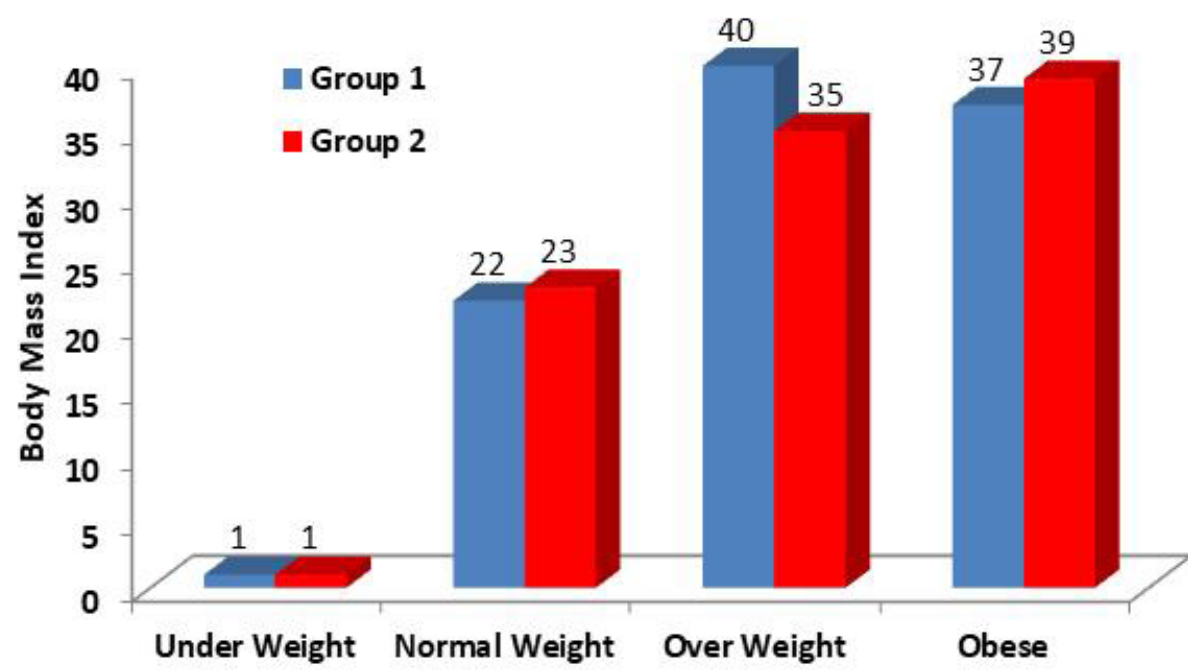

Figure I Distribution of patients according to BMI in Group I and Group 2.

The mean serum level of prolactin, TSH, FSH, LH, $\mathrm{FT}_{4}, \mathrm{E}_{2}$ and BMI are shown in Table 1. The serum Prolactin concentration was higher in the primary infertile group $(843.15 \pm 5.24$ as compared to that in the control group $(279.36 \pm 10.10)$ and it was statistically highly significant.

Table I Mean \pm SD values of serum hormones and BMI for infertile women (Group I) and fertile women (Group 2). The significant level is $\mathrm{P}<0.05$

\begin{tabular}{llll}
\hline \multirow{2}{*}{ Parameter } & Group I & Group 2 & \\
\cline { 2 - 3 } & High PRL (Mean \pm SD) & Normal PRL (Mean \pm SD) & \\
\hline Prolactin $(\mathrm{mlU} / \mathrm{mL})$ & $843.15 \pm 524.7$ & $279.36 \pm 101.0$ & 0 \\
FSH $(\mathrm{mlU} / \mathrm{mL})$ & $4.86 \pm 2.1$ & $4.91 \pm 1.7$ & 0.847 \\
LH $(\mathrm{mlU} / \mathrm{mL})$ & $5.28 \pm 4.1$ & $5.07 \pm 2.7$ & 0.663 \\
TSH $(\mathrm{mlU} / \mathrm{mL})$ & $4.24 \pm 6.1$ & $1.84 \pm 1.0$ & 0 \\
FT4 $(\mathrm{pmol} / \mathrm{L})$ & $13.77 \pm 8.2$ & $12.39 \pm 2.1$ & 0.272 \\
E2 $(\mathrm{pmol} / \mathrm{L})$ & $165.9 \pm 99.8$ & $156.4 \pm 93.6$ & 0.489 \\
BMI & $28.09 \pm 4.5$ & $29.41 \pm 5.9$ & 0.076 \\
\hline
\end{tabular}


Prolactin antagonizes two hormones which are necessary for ovulation: The Follicle Stimulating Hormone (FSH) and the Gonadotropin Releasing Hormone (GnRH). When the prolactin level is high in the blood (hyper-prolactinaemia), the female will not ovulate and this will result in infertility. This anovulation can also cause irregular menstrual cycles. ${ }^{14}$

In this study we found that approximately $(27 \%)$ hyperprolactinemic women had some kind of menstrual disturbance, while only $(16 \%)$ of the women in the control group had irregular periods. This indicates the effect of hyperprolactinemia on menstrual cycle. There is convergence to Nallusamy and Gracelyn ${ }^{15}$ study in which $24.6 \%$ women had hyperprolactinemia.

Hyperprolactinemia causes infertility because prolactin inhibits the GnRH secretion. When the GnRH secretion is low, the FSH and LH secretions are also low and so they do not stimulate the gamete production and the gonadal steroid synthesis. ${ }^{16,17}$

The women with high prolactin levels had also hypothyroidism which was characterized by high levels of serum TSH. A subclinical hypothyroidism which was associated with hyperprolactinaemia was also observed in some cases.

Hyperprolactinaemia which results from a longstanding primary hypothyroidism has been implicated in ovulatory dysfunctions due to an inadequate corpus luteal progesterone secretion. Also, when the circulating prolactin levels are high, they lead to oligomenorrhoea or amenorrhoea. Kumkum et al., ${ }^{14}$ stated that amenorrhoea occurs in hypothyroidism due to hyperprolactinaemia, which results from a defect in the positive feedback of oestrogen on $\mathrm{LH}$, and because of the suppression of LH and FSH.

Table 1 shows the serum hormone and BMI values for experimental group/infertile women (Group 1) and Control Group/normal women (Group 2). The Mean \pm SD value for prolactin for Group 1 was $843.15 \pm 524.7$ and for control group was $279.36 \pm 101.0 \mathrm{mIU} / \mathrm{mL}$. There was a statistically significant difference between the 2 groups. The value in infertile women was significantly higher $(\mathrm{P}<0.05)$. The high prolactin level is associated with anovulation or may cause infertility directly or indirectly. ${ }^{18}$ The serum levels of FSH for case and control group were $4.86 \pm 2.1$ and $4.91 \pm 1.7 \mathrm{mIU} / \mathrm{mL}$, respectively. There was no significant difference between case study and control group. The serum levels of LH for case and control group were 5.28 \pm 4.1 and $5.07 \pm 2.7 \mathrm{mIU} / \mathrm{mL}$. There was no significant difference between case study and control group. This result opposite with, ${ }^{19}$ who reported significantly lower level of LH and FSH than in the control group.

The serum levels of TSH for study and control groups were $4.24 \pm 6.1$ and $1.84 \pm 1.0 \mathrm{mIU} / \mathrm{mL}$, respectively. There was a significant difference between the study and control group $\mathrm{P}<0.05$ ). The serum levels of FT4 for study and control groups were 13.77 \pm 8.2 and $12.39 \pm 2.1 \mathrm{pmol} / \mathrm{L}$, respectively. There was no significant difference between study and the control group. The study showed that the incidence of hyper-prolactinemia in primary hypothyroidism is notable and this disorder is more common in female with primary hypothyroidism than normal individuals. This prevalence agree with an earlier study that found a high incidence of hyperprolactinemia in infertile women and its positive association with hypothyroidism. ${ }^{21}$

Determination of thyroid function (T3, T4 and TSH) is essential in the initial infertility work up as hypothyroidism can cause anovulation, luteal phase defect, amenorrhea, and alterations in the level of sex hormone-binding globulin, estradiol, testosterone, estrone and androstenedione.
The incidence of PCOS in women with hyperprolactinemia is around $32 \%$ while $24 \%$ women with normal prolactin had PCOS. This indicates some correlation of hyperprolactinemia with PCOS. However, PCOS and hyperprolactinemia can occur independently as well. A previous study ${ }^{21}$ concluded that the incidence of hyperprolactinemia in women with PCOS is around $21 \%$. So here we found that hyperprolactinemia and PCOS are two different entities. And hence, there is need to evaluate women for hyperprolactinemia and thyroid function when presented with menstrual disturbances due to $\mathrm{PCOS}$.

The rate of positive clinical pregnancy in control group was $29 \%$ while in case group 19\%. This indicates that hyperprolactinemia affects the success of IVF. The serum levels of $E_{2}$ for case and control groups were $165.9 \pm 99.8$ and $156.4 \pm 93.6 \mathrm{pmol} / \mathrm{L}$, respectively. There was no significant difference between study and control groups.

\section{Conclusion}

The results of this study showed that the serum prolactin and TSH are significantly higher in infertile Saudi women as compared to those fertile Saudi women in the control group. It is, therefore, important to include determination of these hormones in the initial investigation work up of Saudi women seeking infertility treatment.

\section{Acknowledgments}

None.

\section{Funding}

None.

\section{Conflicts of interest}

The author declares that there is no conflict of interest regarding this study.

\section{References}

1. Sudha P, Malini MS, Indira N, et al. A study of prolactin levels in infertile women. Journal of Dental and Medical Sciences. 2013;14(10):10-18.

2. Tabong PT, Adongo PB. Infertility and childlessness: a qualitative study of the experiences of infertile couples in North Ghana. BMC Pregnancy Childbirth. 2013;13:72.

3. Abolfotouh MA, Alabdrabalnabi AA, Albacker RB, et al. Knowledge, attitude, and practices of infertility among Saudi couples. International journal of general medicine. 2013;(6):563-573.

4. Sharma R, Biedenharn KR, Fedor JM, et al. Lifestyle factors and reproductive health: taking control of your fertility. Reprod Biol Endocrinol. 2013;11:66.

5. Lee DS, Ryoo NY, Lee SH, et al. Basal luteinizing hormone and follicular stimulating hormone: is it sufficient for the diagnosis of precocious puberty in girls?. Ann Pediatr Endocrinol Metab. 2013;18(4):196-201.

6. Shamali J, Seema V. Hyperprolactinemia and its comparison with hypothyroidism in infertile women. J Clin Diagn Res. 2013;8(2):106-108.

7. Hamoda H, Khalaf Y, Carroll P. Hyperprolactinaemia and female reproductive function: what does the evidence say?. The Obstetrician \& Gynaecologist. 2012;14(2):81-86.

8. Saleem M, Martin H, Coates P. Prolactin biology and laboratory measurement: an update on physiology and current analytical issues. Clin Biochem Rev. 2018;39(1):3.

9. Freeman ME, Kanyicska B, Lerant A, et al. Prolactin: Structure, function, and regulation of secretion. Physiol Rev. 2000;80:1523-1631. 
10. Grattan DR. 60 years of neuroendocrinology. The hypothalamo-prolactin axis. J Endocrinol. 2015;226:101-122.

11. VanKlompenberg MK, Manjarin R, Donovan CE, et al. Regulation and localization of vascular endothelial growth factor within the mammary glands during the transition from late gestation to lactation. Domest Anim Endocrinol. 2016;54:37-47.

12. Shoupe D, Mishell DR. Hypoprolactinemia: Diagnosis and treatment. Mishell's textbook of Infertility, Contraception and Reproductive Endocrinology. 1997;4:323-341.

13. Kabel AM. Polycystic ovarian syndrome: insights into pathogenesis, diagnosis, prognosis, pharmacological and non-pharmacological treatment. J Pharma Reports. 2016;1:103.

14. Kumkum A, Jasmine K, Shweta G, et al. Hyperprolactinema and its correlation with hypothyroidism in infertile women. Obstet Gynecol India. 2006;56:68-71.

15. Nallusamy S, Gracelyn LJ. Prevalence of hyperprolactinemia in infertile women and its association with hypothyroidism. Int $J A d v$ Med. 2016;3:33-38.
16. Koutras DA. Disturbances of menstruation in thyroid disease. Ann NY Acad Sci. 1997;816:280-284.

17. Poppe K, Velkeniers B. Thyroid and infertility. Verh K Acad Geneeskd Belg. 2002;64(6):389-399.

18. Idrisa A, Kawuwa MB, Habu SA, et al. Prolactin levels among infertile women in Maiduguri, Nigeria. Tropical Journal of Obstetrics and Gynaecology. 2003;20(2):97-100.

19. Ahmed K, Ayman A, Mohamed S, et al. Changes in serum prolactin level during Intracytoplasmic sperm injection, and effect on clinical pregnancy rate: a prospective observational study. BMC Pregnancy and Childbirth. 2018;18:141.

20. Sharma N, Baliarsingh S, Kaushik GG. Biochemical association of hyperprolactinemia with hypothyroidism in infertile women. Clin Lab. 2012;58(7-8):805-810.

21. Swathi T, Sujaya V Rao. Prevalance of hyperprolactinemia in PCOS International Journal of Science and Research (IJSR). 2015;6(11):391. 